\title{
The Inn at the Crossroads - A Model of Distributed Spatial Knowledge
}

\author{
Ioan SUSNEA \\ University "Dunarea de Jos" of Galati, \\ 47 Domneasca St., Galati, 800008, Romania \\ ioan.susnea@ugal.ro
}

\begin{abstract}
The paper describes a simple experiment aimed to demonstrate the possibility to create distributed cognitive maps of certain environments by means of recording basic information about the purposeful motion of a population of mobile agents. The environment is assumed to consist in a set of behaviorally significant places interconnected by predefined paths, just like the inns at the crossroads used to be deployed along and across populated areas centuries ago. These active places interact with the mobile agents and create local patches of relevant navigation information, which is shared with other agents that reach the respective place. As a result, the system exhibits a globally consistent spatial behavior, using only locally available, incomplete spatial knowledge. A NetLogo simulation allowed us to compare the average distance traveled by the agents, and the average travel time before reaching their goals, with a similar system wherein the agents walk randomly. As expected, the agents using the distributed spatial knowledge reached their goals much faster than those walking randomly in the same environment. We also explore the possible applications of systems built according to the principles described here.
\end{abstract}

Keywords: Multi-agent systems, spatial cognition, distributed memory maps, cognitive stigmergy

\section{Introduction}

Arguably, spatial orientation is one of the most important requirements for survival in the biological world. Most living organisms need to be able to navigate through the environment in search of food, to return to certain significant places such as nests or shelters, or to recognize certain "signs" in the environment as potential dangers or hideouts.

Animals often exhibit amazing abilities of spatial navigation: consider the examples of the Chinook salmon (oncorhynchus tshawytscha) that travel $3000 \mathrm{Km}$ to spawn in the exact place where they come to life, or of the Arctic tern (sterna paradisaea) that fly $70,000 \mathrm{Km}$ each year, from Greenland to Antarctica and back.

The neural mechanisms that allow such extraordinary exploits are not fully understood. After studying how rats find their way to the food in a maze, Tolman [1] coined the term "cognitive maps" to designate some hypothetical neural structures that reproduce geometric features and relative positions of the perceived objects in space.

Subsequent studies of O'Keefe \& Dostrovsky [2], Ranck [3], and Moser [4] and many others support the hypothesis of Tolman, by providing experimental evidence of the existence of specialized neurons called "place cells", "head direction cells", and "grid cells" (in hippocampus and in other areas of the brain), all involved in the process of spatial orientation.

Though the formal definition of the cognitive maps is still under debate (see [5], [6], [7]), it is now widely accepted that there exists a neural model of the spatial environment, and in a general sense, a cognitive map is any mental representation of the space.

The interest for spatial cognition and mapping exceeds the boundaries of the biology and neurology, and there is now a huge literature that explores these topics from various perspectives: psychology, engineering (robotics, wireless sensor networks), urbanism, etc.

In engineering, for obvious reasons, the problems related to spatial localization and mapping were mainly studied in the context of robotics (see [8], [9], [10], [11]] for a quick image of the complexity of these problems).

An interesting taxonomy of the approaches used in robotic mapping starting from the hierarchy of the levels of abstraction of the features used in navigation is available in [12].

Rather surprisingly, while reviewing the vast literature dedicated to spatial cognition from an engineering perspective, it becomes obvious that there are at least two implicit assumptions that tend to hinder the understanding of these topics: 
- One is the idea that the spatial cognition is a purely individual process, wherein the environment is entirely passive - just the object of the cognitive reflection, and the reflexive subject is completely disconnected from other individuals.

- The second implicit assumption is the idea that "the map is not the territory" - a famous statement that seems so obvious that makes us ignore the fact that the territory often contains map-specific features, usually in the form of traces resulted from the activity of other agents. These traces may be interpreted as indirect communication messages capable to influence the spatial navigation of the other agents (see [13]). In fact, the boundary between the map and the territory is not always very crisp.

The aim of the research described in this paper is to explore the situations when the spatial cognition is neither individual nor local, i.e. when the cognitive map results from the activity of a whole population of agents, and this map is organized as a set of "sub-maps" distributed over the environment in certain active places that act as "patches of knowledge".

To this purpose, we have created a NetLogo ([14]) model, wherein a number of mobile agents move through an environment, organized as a topological graph, consisting in a set of nodes, interconnected by edges.

However, this graph structure only exists for an external observer. No instance in this system has a global representation of the topology of the environment, and the mobile agents ("walkers") navigate relying only on the incomplete and locally available information resulted from their interaction with the nodes of the graph.

The NetLogo simulation allowed us to measure and compare the average distance traveled by the agents, and the average travel time before reaching their goals, with those measured in a similar system wherein the agents walk randomly. As expected, the agents using the distributed cognitive map reached their goals up to ten times faster than those walking randomly in the same environment. We also explored a few possible applications of systems built according to the principles described here.

The rest of this paper is structured as follows:
- Section 2 contains a brief review of the related work;

- Section 3 contains a description of the experiment, and the results.

- Section 4 is reserved for discussion and conclusions.

\section{Related Work}

The idea that the processes related to cognition may transcend the individual agents surfaced with the advent of the concept of "swarm intelligence" ([15]). Chialvo \& Millonas in [16] were the first to explicitly talk about cognitive maps with respect to the activity of the swarms.

Ricci et al. in [17] go beyond the collectively acquired spatial cognition, and propose the more general concept of "cognitive stigmergy" - a concept that was later explored from multiple practical perspectives, e.g. for group decision support systems ([18]), stigmergic learning ([19]), and many others (see [20]).

The importance of the environment as mediator of the (cognitive) interactions between agents in multi-agent systems (MAS) was stressed among others - by Susi \& Ziemke (21]) and Parunak ([22]).

Omicini (in [23]) uses a suggestive metaphor ("agents writing on walls") to describe the indirect communication between agents in cognitive MAS.

The studies about spatially distributed cognitive maps are far less common. One of the most significant works, from the perspective of this presentation, is [24], which is actually the seminal work that inspired the present research.

In [24], Conradt proposes a system wherein a network of behaviorally significant places (called "place agents", PAs), are capable to communicate with their direct neighbors, and to control a mobile robot that "moves in space and reports sensor perceptions from various places". "Each of these PAs is unaware of its position within the network and the position it represents in global space. The topology of the network - which reflects the structure of traversable external space - only exists implicitly."

Rather than using a Cartesian representation of the environment (see Fig. 1a) as in [10], or a topological map (Fig.1b) as in [12], the robot in [24] creates and uses a distributed cognitive 
map (Figure 1c, 1d) consisting in a set of smaller maps, each describing the geographical area surrounding the active places. The sensing and processing tasks required for creating these maps are also distributed between the robot and the PAs.

a

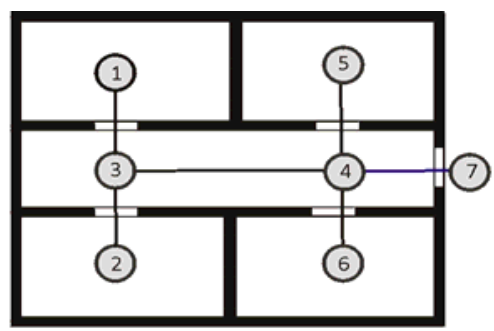

b
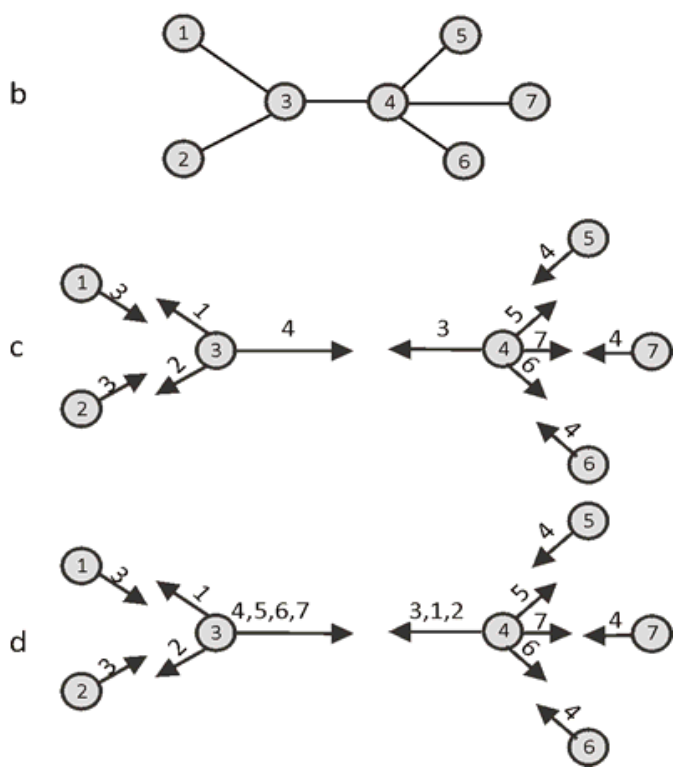

Figure 1. Various methods to represent the space: a) as a Cartesian map; b) as a topological graph; c) and d) as a collection of data structures located in different places

According to Conradt, "this simple strategy significantly reduces computational complexity, is robust to local perturbations, scales well with the size of the navigable region, and permits a robot to autonomously explore, learn, and navigate large unknown environments".

Enough reasons for a closer look at this solution. Which we actually did in the present study.

\section{Description of the Experiment}

The conceptual model that best describes the experiment presented here is the metaphor of the inns at the crossroads - those places where the travellers used to exchange information about the state of the roads, and the available routes to their destinations.
Consider a space consisting in a finite set of "places" (the inns - represented as circles in Figure 2), interconnected by a number of "road segments".

Obviously, this space can be mathematically represented by a graph, wherein the "places" are the vertices of the graph ("nodes" according to the terminology of NetLogo), and the roads are the edges of the graph (called "links" in NetLogo).

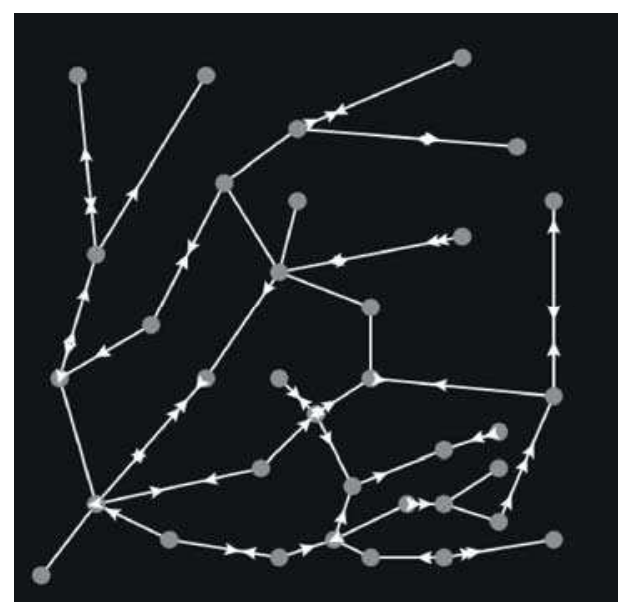

Figure 2. A snapshot of the graph-like NetLogo environment.

A "route" in this space is any sequence of adjacent edges, connecting an arbitrary pair of nodes.

Assume that this space is traveled by $\mathrm{N}$ mobile agents (for simplicity, assume that all the travelers move with the same speed).

Each traveler knows his goal, and the origin of his journey (the node where the trip started), but does not know any route that connects the starting point to the goal, and is unaware of his current position. Moreover, travelers do not have memory - they can only remember the latest segment of road they have used, and they cannot directly communicate with each other.

Similarly, the "inns" located in the nodes of the graph do not have any a-priori knowledge about the structure of the graph: they only own a list of their adjacent nodes (see Figure 1c).

Remember that the graph depicted in Figure 2 only exists for an external observer: neither the travelers, nor the nodes do not have knowledge about the global structure of the graph representing their environment.

In order to allow the indirect communication between the mobile agents, all the inns own a data structure that can be annotated by the 
travelers (a sort of blackboard, where the travelers can write) by adding information about where they come from, and what was the last segment of road they traveled before reaching the current node (see Figure 1d).

This information is later used by the other agents that reach the inn to select from the adjacent segments of road available at the current node, that segment previously used by a traveler coming from where they intend to go. See Figure 3 for a detailed description of the behavior of the mobile agents.

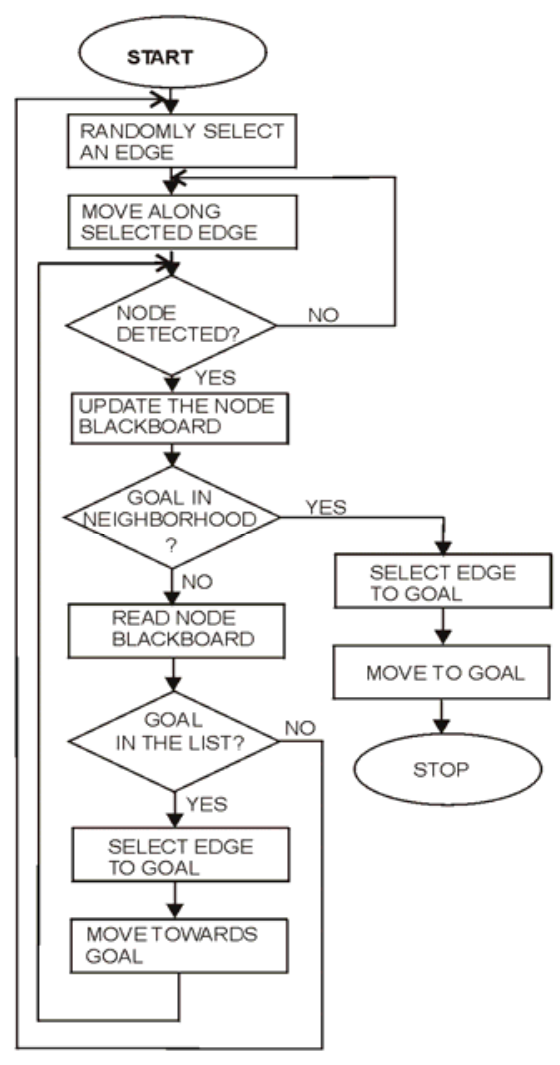

Figure 3. A flowchart describing the behavior of one of the mobile agents

If more that one of the adjacent edges is indicated as possible road to the goal, the traveler randomly selects one of available roads.

If the graph is fully connected (as the one depicted in Figure 2), all the travellers will eventually reach their goals, even if they randomly select the next segment of road at every node (see [25]).

Therefore, we conducted simulations for two different scenarios:

- In the first scenario, the agents select the road segments in a purely random way;
- In the second scenario, the agents have the behavior described in Figure 3.

The number of travelers $\mathrm{N}$ was variable, in the interval $[1,200]$. For each value of $\mathrm{N}$, we measured the average distance traveled by the agents before reaching their goal, and the average travel time, in both of the above mentioned scenarios.

The results are presented in Figures 4-6. Figure 4 shows the values of the average time and distance for the random walk scenario, while Figures 5 and 6 show the average travel time, and average distance traveled to goal when using the distributed cognitive map (DCM).

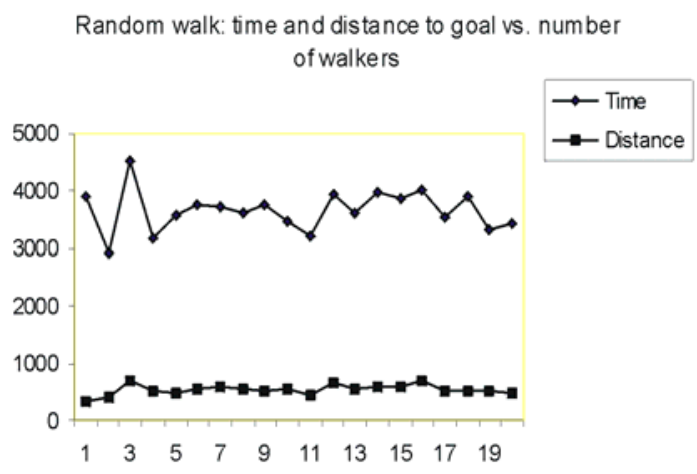

Figure 4. The performances of the agents in the random walk scenario

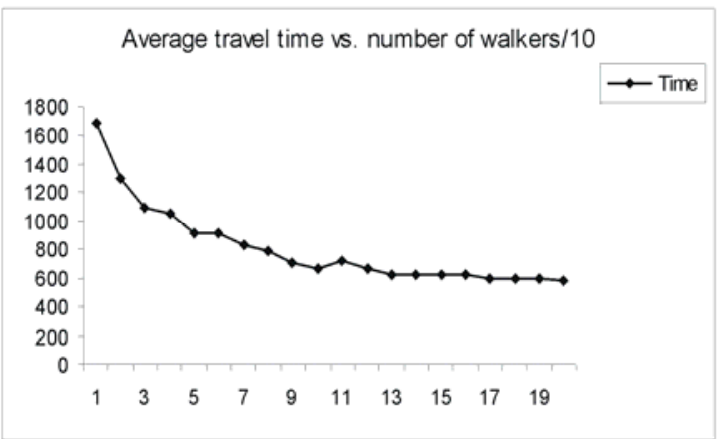

Figure 5. The average travel time (measured in NetLogo ticks) when using the DCM

As expected, in the random walk scenario, the number of travelers has no influence on the average travel time and distance, since the agents are totally disconnected from each other.

On the contrary, in the DCM scenario, the number of agents is a measure of the "stigmergic coupling" between the agents, and its influence is quite visible: the average traveled distance is up to ten times smaller than the values recorded in the random walk scenario. 


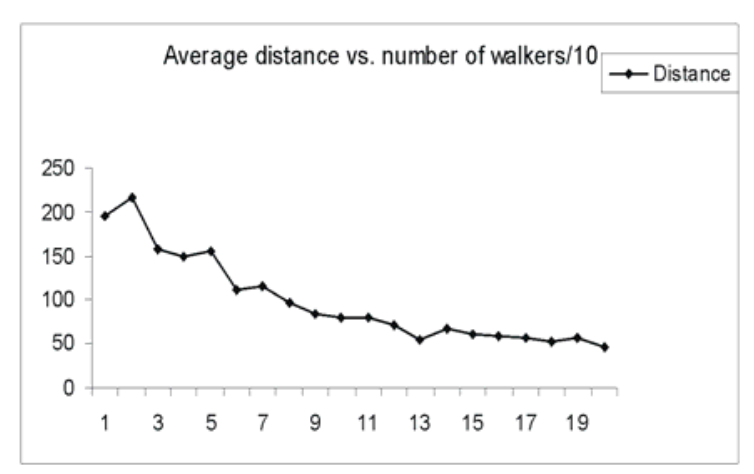

Figure 6. The average distance traveled by the agents (measured in NetLogo units) when using the DCM

\section{Discussion and Conclusion}

The simple experiment described above is aimed to demonstrate that simple protocols of information sharing may lead to creating interesting "patches of collective knowledge", with possibly useful applications.

Among these possible applications, we could count:

- A system for guiding visually impaired people in supermarkets, and in the cities. Such a system would consist in a number of relatively low cost devices deployed along the main pathways, which could implement the data exchange protocols described above, by communicating with the user's smartphones via Bluetooth (see also [26] for related solutions and details).

- A similar system of "electronic blackboards" mounted along the roads and designed to communicate with the passing vehicles may provide useful navigation information and even implement "customized road signaling".

- The proposed design approach could drastically cut the costs for manufacturing a class of service robots, like those operating in maze-like environments (hospitals, warehouses, etc.)

- Finally, the principles of creating DCMs described here may serve for coordinating the navigation in virtual environments, like those used in multi-player games.

Considering the above (obviously incomplete) list of possible applications, we conclude that the proposed solution for creating distributed cognitive maps is definitely worth further attention.

\section{Acknowledgements}

This research was partly funded by the DAAD grant nr. 91574298, in the programme "Research Stays for Academics and Scientists 2015" (50015559).

The author gratefully acknowledges the support of Prof. Jörg Conradt, and of Cristian Axenie of the Neuroscientific System Theory Group, within the Technical University of Munich, who made this research possible.

\section{REFERENCES}

1. TOlman, E. C., Cognitive Maps in Rats and Men, Psychological Review, 1948, 55.4: 189.

2. O'KEEFE, J., DOSTROVSKY, J., The Hippocampus as a Spatial Map. Preliminary Evidence from Unit Activity in the Freely-Moving Rat, Brain Research, 1971, 34.1: 171-175.

3. RANCK JR., J. B. Head-direction Cells in the Deep Cell Layers of Dorsal Presubiculum in Freely Moving Rats, Society for Neuroscience Abstracts, 1984.

4. MOSER, E. I., E., KROPFF, M.-B., MOSER, Place Cells, Grid Cells, and the Brain's Spatial Representation System, Neuroscience, 2008, 31.1: 69.

5. BENNETT, A. T., Do Animals Have Cognitive Maps?, The Journal of Experimental Biology, 1996, 199.1: 219-224.

6. JACOBS, L. F., The Evolution of the Cognitive Map, Brain, Behavior and Evolution, 2003, 62.2: 128-139.

7. CHEESEMAN, J. F., et al. Way-finding in Displaced Clock-shifted Bees Proves Bees Use a Cognitive Map, Proceedings of the National Academy of Sciences, 2014, 111.24: 8949-8954.

8. THRUN, S., et al. Robotic Mapping: A Survey. Exploring Artificial Intelligence in the New Millennium, 2002, pp. 1-35.

9. VASUdeVAn, S., et al. Cognitive Maps for Mobile Robots - an Object Based Approach. Robotics and Autonomous Systems, 2007, 55.5: 359-371.

10. WOLF, D. F., SUKHATME, G. S., Mobile Robot Simultaneous Localization and 
Mapping in Dynamic Environments, Autonomous Robots, 2005, 19.1: 53-65.

11. GARCIA-FIDALGO, E.; A., ORTIZ, Vision-based Topological Mapping and Localization Methods: A Survey, Robotics and Autonomous Systems, 2015, 64: $1-20$.

12. TAPUS ,A., Topological SLAMSimultaneous Localization and Mapping with Fingerprints of Places, 2005, PhD Thesis, Université Joseph Fourier Grenoble, France.

13. TUMMOLINI, L., CASTELFRANCHI, C., Trace Signals: the Meanings of Stigmergy, Environments for Multi-Agent Systems III, Springer Berlin Heidelberg, 2007, pp. 141-156.

14. WILENSKY, U., NetLogo (and NetLogo User Manual), Center for Connected Learning and Computer-Based Modeling, Northwestern University. http://ccl. northwestern. edu/netlogo, 1999.

15. BENI, G., J., WANG, Swarm Intelligence in Cellular Robotic Systems, Robots and Biological Systems: Towards a New Bionics?, Springer Berlin Heidelberg, 1993, pp. 703-712.

16. CHIALVO, D. R., M. M., MiLlONAS, How Swarms Build Cognitive Maps, The Biology and Technology of Intelligent Autonomous Agents, Springer Berlin Heidelberg, 1995, pp. 439-450.

17. RICCI, A., et al., Cognitive Stigmergy: Towards a Framework Based on Agents and Artifacts, Environments for Multi-Agent Systems III, Springer Berlin Heidelberg, 2007. pp. 124-140.

18. ZAMFIRESCU, C.-B., FILIP, F. G., Swarming Models for Facilitating Collaborative Decisions, International Journal of Computers, Communications \& Control, 2010, 1: 1841-1844.
19. SUSNEA, I., G., VASILIU, D. E., MITU, Enabling Self-Organization of the Educational Content in Ad Hoc Learning Networks, Studies in Informatics and Control, 2013, 22.2: 143-152.

20. SUSNEA, I., Engineering Human Stigmergy. International Journal of Computers Communications \& Control, 2015, 10.3: 420-427.

21. SUSI, T., T., ZIEMKE, Social Cognition, Artefacts, and Stigmergy:

A Comparative Analysis of Theoretical Frameworks for the Understanding of Artefact-Mediated Collaborative Activity. Cognitive Systems Research, 2001, 2.4: 273-290.

22. PARUNAK, H. V. D., A Survey of Environments and Mechanisms for Human-Human Stigmergy, Environments for Multi-Agent Systems II. Springer Berlin Heidelberg, 2006. pp. 163-186.

23. OMICINI, A., Agents Writing on Walls: Cognitive Stigmergy and Beyond, in The Goals of Cognition. Essays in Honor of Cristiano Castelfranchi, vol. 20 of Tributes, chapter 29, 543-556, College Publications, London, (December 2012).

24. CONRADT, J.-A., A Distributed Cognitive Map for Spatial Navigation Based on Graphically Organized Place Agents, 2008, PhD Thesis, Swiss Federal Institute of Technology, Zurich.

25. LOVÁSZ, L., et al., Random Walks on Graphs: A Survey, Combinatorics, Paul Erdos is Eighty, 1996, 2: 353-398.

26. BALATA, J., et al., Collaborative Navigation of Visually Impaired. Journal on Multimodal User Interfaces, 2014, 8.2: $175-185$ 